\title{
Exploration of Boletes from Xiangtoushan National Nature Reserve, Guangdong Province, China
}

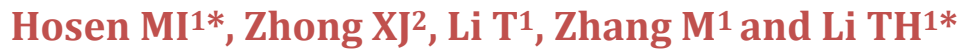 \\ ${ }^{1}$ State Key Laboratory of Applied Microbiology Southern China, Guangdong Provincial \\ Key Laboratory of Microbial Culture Collection and Application, Guangdong Institute of \\ Microbiology, Guangzhou 510070, China \\ ${ }^{2}$ Administration of Guangdong Xiangtoushan National Nature Reserve, Huizhou- \\ 516001, Guangdong, China
}

\section{Research Article \\ Volume 1 Issue 1}

Received Date: September 11, 2018

Published Date: October 09, 2018

DOI: 10.23880 /oajmms-16000101

*Corresponding author: Md Iqbal Hosen/Tai-Hui Li, State Key Laboratory of Applied Microbiology Southern China, Guangdong Provincial Key Laboratory of Microbial Culture Collection and Application, Guangdong Institute of Microbiology, Guangzhou 510070, China, Email: iqbalmyco@gmail.com/mycolab@263.net

\section{Abstract}

A first-time survey of boletes from Xiangtoushan National Nature Reserve of Guangdong Province in China was conducted from April 2015 to August 2016. A total of 18 collections of boletes were studied, which represents 16 phylogenetic species in 12 genera, including four species of Tylopilus, two of Aureoboletus, and one each of the genera Austroboletus, Boletus, Boletellus, Chiua, Heimioporus, Pulveroboletus, Parvixerocomus, Rossbeevera, Strobilomyces and Sutorius. Among the 16 species of bolete, six species, namely Aureoboletus tomentosus, Chiua virens, Parvixerocomus aokii, Pulveroboletus brunneoscabrosus, Sutorius eximius and Tylopilus balloui are presented here. The remaining 10 phylogenetic species (one each for Aureoboletus, Austroboletus, Boletus s.l., Boletellus, Heimioporus, Rossbeevera, Strobilomyces and three for Tylopilus) were still not given valid name due to the paucity of collections. Brief morphological descriptions and color photos of the six recognized species are provided.

Keywords: Boletaceae; East Asia; Morphology; Subtropical; Taxonomy

\section{Introduction}

China is extraordinary rich in higher fungi and one of the world's 25 Biodiversity Hotspots [1,2]. Studies on higher fungi from China have been started at the end of $19^{\text {th }}$ or very beginning of the $20^{\text {th }}$ century $[2,3]$. Since then, over 10,000 fungal species have been documented from the vast territory of China [2]. Among them, 3,725 species belong to Basidiomycota [2]. After that, many new records, new genera and species of fungi have been reported from China and many more yet need to be documented.
It is well-known from the literatures that most of the fungal species are laying in the tropic areas. Xiangtoushan National Nature Reserve (dominated by evergreen broadleaved trees) is located in the subtropical area of Guangdong Province of China. However, prevalence of basidiomycete fungi in Xiangtoushan National Nature Reserve (XNNR) is poorly studied. This is the first survey of basidiomycete fungi from XNNR, and this study is mainly focused on boletes.

Boletes are the members of the family Boletaceae and conspicuous components in nature which plays an 
important role in forests ecosystem. Boletes are mainly recognized by its poroid hymenophore except Phylloporus Quél. and few gasteroid boletes. China is an extraordinary rich in the diversity and distribution of boletes. Since midnineteen century ca. 500 boletes species have been reported from this country [4-42]. Based on integration morphological data along with molecular-phylogenetic evidence, Boletaceae have yielded seven subfamilies, 62 generic clades (excluding some gasteroid bolete genera) of which 52 are occurred in China based on multi-locus gene phylogenetic analyses $[40,41]$.

Prior to this study only a single species, namely Tricholoma sinoacerbum T.H. Li, Iqbal Hosen \& Ting Li has been reported so far [43]. Preliminary investigation seems that this region harbors many interesting and undescribed fungi which need to be documented for better understanding the systematics of macrofungi. The aims of this study are-i) to investigate the boletes fungal resources in this region, ii) to identify the boletes at the generic/species level based on morphological aspect.

\section{Materials and Methods}

\section{Description of the Study Site}

Xiangtoushan National Nature Reserve is located $\left(23^{\circ}\right.$

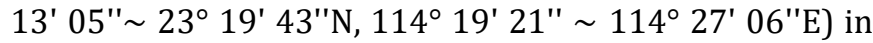
Boluo Country, North Huizhou, Guangdong Province, China which covers about 10,696.9 hectares [44]. It was established in 1998 and granted "National Reserve" status in 2002 [44]. It has been established in order to protect subtropical broad-leaved evergreen forest and rare wildlife. The reserve has a rich biodiversity, a total number of plants 1646 (including gymnosperm and angiosperm) and numbers of animals 305 (including birds, reptiles, etc.) have been recorded so far [44]. However, little is known about the fungal diversity from this habitat.

\section{Sample Collections and Deposition}

Fungal samples were collected from XNNR of Guangdong Province in China. All samples were deposited in the Fungal Herbarium of Guangdong Institute of Microbiology (GDGM), Guangzhou, China.

\section{Morphological Studies}

Morphological studies include field notes and microscopic observations. A small piece of dried specimen was revived in $\mathrm{H}_{2} \mathrm{O}, 5 \% \mathrm{KOH}$ and Congo red. The notation $[\mathrm{n} / \mathrm{m} / \mathrm{p}]$ is used in the descriptions of basidiospore measurements, which means $n$ basidiospores from $m$ basidiomata of $p$ collections were measured; 20 basidiospores were measured from the collected material. Dimension for basidiospores are given as $(a-) b-c(-d)$, in which ' $b-c$ ' contains a minimum of $90 \%$ of the measured values and extreme values ' $a$ ' and ' $d$ ' are given in parentheses. $Q_{m}=Q \pm S D$ : $Q$ indicates the length/width ratio of a measured basidiospore, $\mathrm{Q}_{\mathrm{m}}$ indicates to the average of $\mathrm{Q}$ basidiospores and $\mathrm{SD}$ is the standard deviation. Radial-vertical sections were made halfway of the pileus and near to the disc of the pileus for the pileipellis observations. Line drawings were done free hand.

\section{Results}

This is the first-time survey of boletes fungal resources from XNNR of Guangdong Province in China. Approximately, 18 collections of boletes were made from that habitat and 16 phylogenetic species were identified. Among the collections, six species were documented with brief description and the remaining 10 phylogenetic species were still not given valid name due to the paucity of collections. A summary of the identified species is presented in Table 1.

\begin{tabular}{|c|c|}
\hline Name of the species & $\begin{array}{c}\text { Voucher specimen } \\
\text { number }\end{array}$ \\
\hline Aureoboletus sp. & GDGM 44479 \\
\hline $\begin{array}{c}\text { Aureoboletus tomentosus G. Wu \& } \\
\text { Zhu L. Yang }\end{array}$ & GDGM 44727 \\
\hline Austroboletus sp. & GDGM 44717 \\
\hline Boletellus sp. & GDGM 44726 \\
\hline Boletus sp. & GDGM 44985 \\
\hline $\begin{array}{c}\text { Chiua virens (W.F. Chiu) Yan C. Li } \\
\text { \& Zhu L. Yang }\end{array}$ & $\begin{array}{l}\text { GDGM } 44483 \\
\text { GDGM } 46060\end{array}$ \\
\hline Heimioporus sp. & GDGM 44988 \\
\hline $\begin{array}{l}\text { Parvixerocomus aokii G. Wu, N.K. } \\
\text { Zeng \& Zhu L. Yang }\end{array}$ & $\begin{array}{l}\text { GDGM } 45163 \\
\text { GDGM } 46620\end{array}$ \\
\hline $\begin{array}{l}\text { Pulveroboletus brunneoscabrosus } \\
\text { Har. Takah. }\end{array}$ & GDGM 45147 \\
\hline Rossbeevera sp. & GDGM 45266 \\
\hline Strobilomyces sp. & GDGM 49208 \\
\hline $\begin{array}{l}\text { Sutorius eximius (Peck) Halling, M. } \\
\text { Nuhn \& Osmundson }\end{array}$ & GDGM 44997 \\
\hline Tylopilus balloui (Peck) Singer & GDGM 44989 \\
\hline Tylopilus sp.1 & GDGM 46621 \\
\hline Tylopilus sp.2 & GDGM 44768 \\
\hline Tylopilus sp. 3 & GDGM 45050 \\
\hline
\end{tabular}

Table 1: Name of identified bolete genera/species of Boletaceae and their voucher information from XNNR, Guangdong Province, China. 


\section{Open Access Journal of Mycology \& Mycological Sciences}

\section{Taxonomy}

Aureoboletus tomentosus G. Wu \& Zhu L. Yang, Fungal Diversity 81: 51 (2016) Figure 1a

Basidiomata small to medium-sized. Pileus $40 \mathrm{~mm}$ broad, applanate, golden yellow to maize yellow, scurfy, tomentose, surface dry. Hymenophore adnate to slightly decurrent; pores angular, yellow to maize yellow, nearly concolorous to pileus. Stipe $60 \times 7 \mathrm{~mm}$, central, cylindrical, slightly thickening toward base, concolorous with pileus or slightly paler; basal mycelium yellowish cream-white.

Basidiospores [20/1/1] 10-12 × 3.8-4.5 $\mu \mathrm{m}[\mathrm{Q}=2.63-$ 2.83(-3.0), $\mathrm{Q}_{\mathrm{m}}=2.81 \pm 0.13$ ], boletoid, cylindro-fusoid to fusoid, yellowish brown to brownish yellow, smooth, inamyloid. Basidia 22-30 × 8-9 $\mu \mathrm{m}$, clavate, 4-spored, sometimes 2-4 spored. Caulocystidia 25-35 × 7-9 $\mu \mathrm{m}$, clavate to fusoid, vacuolar pigmentation, thin walled, smooth. Clamp connections absent.

Known Distribution: Currently known from southern and south western China.

Specimens Examined: CHINA, Guangdong Province, Huizhou, Boluo County, Xiangtoushan National Nature Reserve, 7 Jul 2015, Ting Li \& Hao Huang (GDGM 44727).

Commentary: This species has recently been described from southeastern China [41]. It can be easily recognized in the field by its maize yellow to dark yellow basidiomata with adnate to subdecurrent tubes. Phylogenetically, it is a member of the subfamily Xerocomoideae [41].

Chiua virens (W.F. Chiu) Yan C. Li \& Zhu L. Yang, Fungal Diversity 81: 79 (2016) Figure 1b

Basidiomata epigeous, small to medium-sized. Pileus 25-40 mm broad, hemispherical to convex, applanate with age; surface dry, green to dark green or olive-green when young, greenish yellow to mustard yellow with age, covered with fibrillose or tomentose squamules, distinct areolate with age; context $7 \mathrm{~mm}$ thick at stipe apex, yellow to bright yellow, unchanged color when injured. Hymenophore tubular, slightly depressed around apex of stipe; pores angular to slightly round, white when young, becoming pale pink to pink when mature; not staining when injured. Stipe 40-60 × 10-15 mm, subcylindrical, attenuated towards base with mucronate base; surface yellow to mustard yellow upper part but bright yellow to chrome yellow at base; context nearly concolorous with pileus, but bright yellow to chrome yellow at the base. Basal mycelia bright yellow to chrome yellow. Taste and odor not recorded.
Basidiospores [20/1/1] 11-12.5 × 4.5-5.5 $\mu \mathrm{m}[\mathrm{Q}=$ $2.33-2.65, \mathrm{Q}_{\mathrm{m}}=2.44 \pm 0.11$ ], boletoid, fusoid, light yellow to brownish yellow, smooth, inamyloid. Basidia 22-20 $\times$ 8-12 $\mu \mathrm{m}$, clavate, 4-spored, sometimes 2-4 spored. Cheilo- and pleurocystidia 25-35 $\times$ 7-9 $\mu \mathrm{m}$, clavate with subacute apex, thin walled, smooth, hyaline. Clamp connections absent.

Habit and Habitat: Gregarious under broad-leaved trees of Castanopsis fissa, C. chinensis, C. fabri and some trees of the plant family Pinaceae.

Known Distribution: Widely distributed in China.

Specimens Examined: CHINA, Guangdong Province, Huizhou, Boluo County, Xiangtoushan National Nature Reserve, 13 May 2015, Iqbal \& Ting Li (GDGM 44483); same location, 22 Apr 2016, Hao Huang \& Ting Li (GDGM 46060).

Commentary: It was well-known as Tylopilus virens (W.F. Chiu) Hongo, however, recent molecular phylogenetic study placed this species into the genus Chiua [41].

Parvixerocomus aokii (Hongo) G. Wu, N.K. Zeng \& Zhu L. Yang, Fungal Diversity 81: 12 (2016) Figure 1c

Basidiomata epigeous, tiny to small-sized. Pileus 15-20 $\mathrm{mm}$ broad, at first hemispherical, becoming convex to nearly applanate with age; margin slightly incurved; surface dry, smooth, orange red, vivid red to red gradually fading with age or from heavily rain; pileus context 2-3 $\mathrm{mm}$ thick at stipe, pale yellow to light yellow, staining slightly blue when cut. Hymenophore tubular, adnexed to slightly decurrent, light yellow to yellow; tubes 3-4 mm long, nearly round or slightly angular, staining blue to dark blue when injured. Stipe 28-35 × 2-3 mm, central, cylindrical, attenuated towards base, orange red to carrot red, with yellow tinged at the base; context solid, staining slightly blue when cut, solid. Basal mycelium white. Odor and taste not recorded.

Basidiospores [20/1/1] 9-11(-12) × 4-4.5 $\mu \mathrm{m}[\mathrm{Q}=$ (2.20) 2.33-2.43, $\left.\mathrm{Q}_{\mathrm{m}}=2.41 \pm 0.10\right]$, boletoid, cylindrofusoid to narrowly fusoid, light yellow to brownish yellow, smooth, inamyloid. Basidia 24-28(-32) × 8-10 $\mu \mathrm{m}$, clavate, 4-spored, sometimes 2-4 spored with sterigmata up to $5 \mu \mathrm{m}$ high. Cheilo- and pleurocystidia $40-$ $50 \times 11-15 \mu \mathrm{m}$, mostly fusoid with subacute apex to clavate, often lanceolate, thin walled, smooth, hyaline. Clamp connections absent. 
Habitat: Scattered on the ground under broad-leaved trees of Castanopsis fissa, C. chinensis, C. fabri and some other broad-leaved trees.

Distribution: Currently known from south China and Japan [31].

Specimens Examined: CHINA, Guangdong Province, Huizhou, Boluo County, Xiangtoushan National Nature Reserve, 13 May 2015, Iqbal \& Ting Li (GDGM 46620); same location, 7 Jul 2015, Hao Huang \& Ting Li (GDGM 45163).

Commentary: This species was originally described from Japan as Boletus aokii Hongo, but the recent molecular approaches make it as an independent genus in Boletaceae subfamily Boletoideae [41-43].

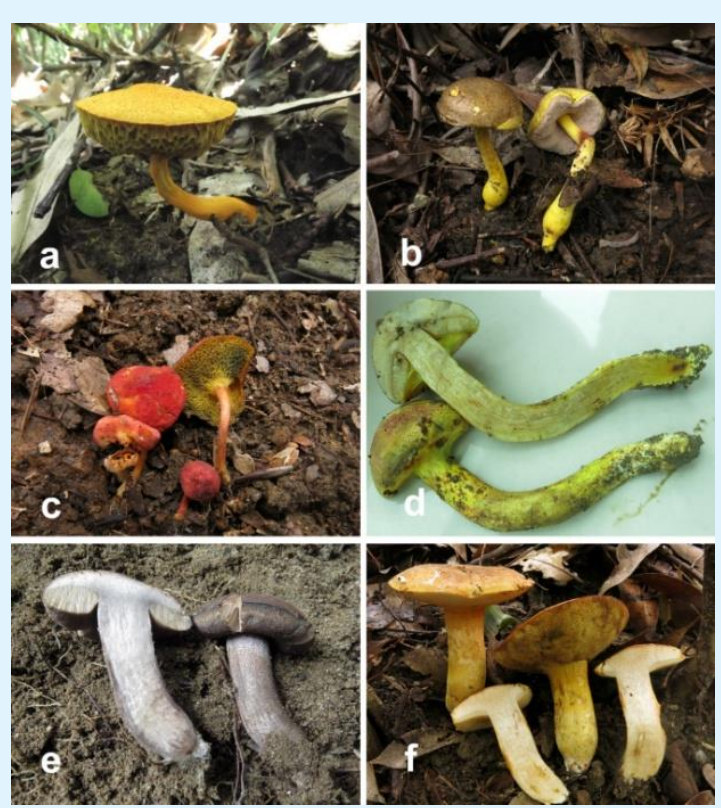

Figure 1: Basidiomata of boletes. a: Aureoboletus tomentosus (GDGM 44727); b: Chiua virens (GDGM 46620); c: Parvixerocomus aokii (GDGM 46620); d: Pulveroboletus brunneoscabrosus (GDGM 45147); e: Sutorius eximius (GDGM 44997); f: Tylopilus balloui (GDGM 44989).

Pulveroboletus brunneoscabrosus Har. Takah., Mycoscience 48(2): 93 (2007) Figure 1d

Basidiomata epigeous, medium-sized. Pileus 30-45 $\mathrm{mm}$ broad, at first hemispherical, becoming convex to nearly applanate with age; marginal veils cottony or cortina (up to $13 \mathrm{~mm}$ wide) that covered young basidiomata, becoming tear off and leaving some remnants near to the edge of margin; surface dry to slightly viscid when wet, covered with orange red, vivid red to reddish brown scales or squamules that gradually fading with age or from heavily rain, background vivid yellow to yellow, turning pale blue when touched or injured; pileus context $11 \mathrm{~mm}$ thick at pileus center, pale yellow to light yellow, staining pale blue slowly in some areas when cut. Hymenophore tubular, free and distinct gutter around the stipe, light yellow to amber yellow; tubes 2-4 mm long, nearly round or slightly angular, staining pale blue very slowly when injured. Stipe 90-105 $\times$ 8-12 mm, central, cylindrical, slightly attenuated towards base, nearly concolorous with pileus but less reddish brown tinged or squamules in the lower half; context pale yellow to pastel yellow, solid, unchanged to hardly changed in pale blue when cut. Basal mycelium pale yellow to dull white. Odor and taste not recorded.

Basidiospores [20/1/1] 7.5-8.5 (-9.5) × 4.4-5 $\mu \mathrm{m}[\mathrm{Q}=$ (1.68-) 1.73-1.92(-2.0), $\left.\mathrm{Q}_{\mathrm{m}}=1.82 \pm 0.09\right]$, boletoid, apex usually tapering, light yellow to brownish yellow, smooth, inamyloid. Basidia 28-35 × 8-11 $\mu \mathrm{m}$, clavate, 4-spored, sometimes 2-4 spored with sterigmata up to $5 \mu \mathrm{m}$ high.

Habitat: Scattered on the ground under broad-leaved trees of Castanopsis fissa, C. chinensis, C. fabri and some other broad-leaved trees.

Distribution: Currently known from south China and Japan [45].

Specimens Examined: CHINA, Guangdong Province, Huizhou, Boluo County, Xiangtoushan National Nature Reserve, 13 May 2015, Iqbal 838 (GDGM 45147).

Commentary: Pulveroboletus brunneoscabrosus was originally described from Japan [45]. Morphologically, this species is somewhat close to P. macrosporus G. Wu \& Zhu L. Yang. However, P. macrosporus differs from $P$. brunneoscabrosus in having orange to orange red scales on the surface and comparatively larger basidiospores 9$12 \times 5-6.5 \mu \mathrm{m}[41]$.

Sutorius eximius (Peck) Halling, M. Nuhn \& Osmundson, Mycologia 104: 955 (2012) Figure 1e

Basidiomata epigeous, medium-sized. Pileus 40-65 mm broad, convex to plano-convex, applanate with age; surface dry, purple brown or violet brown to chocolate brown, concolorous; context $10-15 \mathrm{~mm}$ thick at stipe, not staining when injured. Hymenophore tubular, adnexed, 
depressed around apex of stipe; pores angular to circular pale pinkish to brown; tubes 5-8 $\mathrm{mm}$ long, light brown or brownish purple then dark pinkish brown, not staining when injured. Stipe 40-55 × 15-20 mm, cylindrical, slightly enlarged towards apex, curved, dry, squamules or scabrous throughout surface, with age subpruinose, very pale violet to brownish pink; context solid, white to dull white, not staining, but brownish tinged in some areas when cut. Basal mycelium white. Taste and odor mild.

Basidiospores $[20 / 1 / 1] 10-12 \times 3.8-4.5 \mu \mathrm{m}[\mathrm{Q}=2.63-$ $2.83(-3.0), \mathrm{Q}_{\mathrm{m}}=2.81 \pm 0.13$ ], boletoid, cylindro-fusoid to fusoid, yellowish brown to brownish yellow, smooth, inamyloid. Basidia 22-30 × 8-9 $\mu \mathrm{m}$, clavate, 4-spored, sometimes 2-4 spored. Caulocystidia 25-35 × 7-9 $\mu \mathrm{m}$, clavate to fusoid, vacuolar pigmentation, thin walled, smooth. Clamp connections absent.

Habit and Habitat: Scattered on the ground under broad-leaved trees of Castanopsis fissa, C. chinensis, C. fabri and some other broad-leaved trees.

Known Distribution: Widely distributed and known from North and Central America, East Asia and Southeast Asia.

Specimens Examined: CHINA, Guangdong Province, Huizhou, Boluo County, Xiangtoushan National Nature Reserve, 13 May 2015, Iqbal \& Ting Li (GDGM 44997).

Commentary: Sutorius eximius has been placed in different genera due to its stipe morphology and color of spores. Smith and Thiers [46] placed this species in the genus Tylopilus because of pale pinkish color of basidiospore which is consistent of the genus Tylopilus. However, Singer [47] placed it in the genus Leccinum due to its dark scabrous stipe surface. Recent molecular study suggests that this species represents a unique generic clade in the Boletaceae [48]. This collection has comparatively shorter and narrower basidiospores with lower $Q$ values than recently reported Chinese $S$. eximius (14-15.5 × 4.5-5.5 $\mu \mathrm{m} ; \mathrm{Q}=2.73-3.57)[41]$, suggesting that this species might be another species/variety of this genus. Additional collections and molecular data are needed for the interpretation of this species.

Tylopilus balloui (Peck) Singer, Am. Midl. Nat. 37: 104 (1947) Figure 1f.

Basidiomata epigeous, medium to large-sized. Pileus 40-110 mm broad, hemispherical to convex, applanate with age; surface dry, non-viscid, uneven, margin slightly appendiculate, orange-red to reddish brown; context 10-
$15 \mathrm{~mm}$ thick at stipe, yellow to bright yellow, unchanged color when injured. Hymenophore tubular, slightly depressed around apex of stipe; pores 3-5 mm long, 2-3 per $\mathrm{mm}$, radially arranged to angular, buff white to light cream-white when young, becoming light pink to pinkish when mature, sometimes bluish tinged on the pore surface but not color changed when cut or injured. Stipe 50-60 × 10-15 mm, cylindrical, slightly attenuated towards base; surface pale yellow or light yellow; context dull white to light yellowish white, unchanged when injured. Basal mycelia white. Taste and odor not distinctive.

Basidiospores $[20 / 1 / 1] 6-7 \times 3.6-4.2 \mu \mathrm{m}[\mathrm{Q}=1.63-$ 1.75(-1.83), $\left.Q_{m}=1.73 \pm 0.05\right]$, ovoid to ellipsoid, sometimes nearly bean or kidney-shaped with slightly depression at middle, light yellow to brownish yellow, smooth, inamyloid. Basidia 25-35 × 7-9 $\mu \mathrm{m}$, clavate, 4spored, sometimes 2-4 spored. Cheilo- and pleurocystidia $35-50 \times 7-9 \mu \mathrm{m}$, numerous, mostly fusoid with subacute apex, often lanceolate, thin walled, smooth, yellowish brown vacuolar pigmentation. Pileipellis a trichoderm, composed of yellowish brown pigmented interwoven cylindrical hyphae.

Habit and Habitat: Scattered on the ground under broadleaved trees of Castanopsis fissa, C. chinensis, C. fabri and some other vascular trees.

\section{Distribution: Currently known from East Asia.}

Specimens Examined: CHINA, Guangdong Province, Huizhou, Boluo County, Xiangtoushan National Nature Reserve, 14 May 2015, Iqbal \& Ting Li (GDGM 44989).

Commentary: The Chinese collection T. balloui is characterized by its orange-red to orange-brown pileus, adnate hymenophore, unchanged context, and ellipsoid to ovoid, nearly bean or kidney-shaped in side view with slightly depression at middle of basidiospores. This species is widely distributed and represents a species complex within the subfamily Zangioideae $[20,40,41]$.

\section{Acknowledgements}

This study is supported by the $11^{\text {th }}$ Special Fund of the China Postdoctoral Science Foundation (No. 2018T110854), the NSFC Research Fund for International Young Scientists (No. 31750110476) to the first author $(\mathrm{MIH})$, and the GDAS' Special Project of Science and Technology Development (No. 2017GDAS CX-0825) to the fourth author (MZ). 


\section{Open Access Journal of Mycology \& Mycological Sciences}

\section{References}

1 Myers N, Mittermeier RA, Mittermeier CG, Da Fonseca GA, Kent J (2000) Biodiversity hotspots for conservation priorities. Nature 403(6772): 853-858.

2 Yang ZL (2005) Diversity and biogeography of higher fungi in China. Evolutionary genetics of fungi Horizon Bioscience, Norfolk 2005: 35-62.

3 Patouillard N (1895) Enumeration des champignons recoltes par le rr. pp. Farges et Soulie dans le Thibet oriental \& le Su-Tchuen: L. Declume.

4 Chiu WF (1948) The boletes of Yunnan. Mycologia 40(2): 199-231.

5 Teng SC (1963) Fungi of China: Science Press, Beijing.

6 Yeh KW, Chen ZC (1980) The Boletes of Taiwan (I). Taiwania 25(1): 166-184.

7 Ying J, Ma Q (1985) New taxa and records of the genus Strobilomyces in China. Acta Mycologica Sinica 4(2): 95-102.

8 Zang M (1985) Notes on the Boletales from eastern Himalayas and adjacent of China. Acta Botanica Yunnanica 7: 383-401.

9 Zang M (1986) Notes on the Boletales from eastern Himalayas and adjacent of China (2). Acta Botanica Yunnanica 8: 1-22.

10 Zang M (1992) Sinoboletus, a new genus of Boletaceae from China. Mycotaxon 45: 223-227.

11 Zang M (1996) A contribution to the taxonomy and distribution of the genus Xerocomus from China. Fungal Science 11(1\&2): 1-15.

12 Zang M (1999) An annotated check-list of the genus Boletus and its sections from China. Fungal Science 14: 79-87.

13 Zang M (2006) Flora Fungorum Sinicorum. Boletaceae (I): Science Press, Beijing, 22.

14 Zang M (2013) Flora Fungorum Sinicorum. Boletaceae (II): Science Press, Beijing, 44.

15 Zhang BC, Yu YN (1989) Chamonixia bispora sp. nov. (Boletales) from China. Mycotaxon 35(2): 277-281.
16 Zhishu B, Guoyang Z, Li TH (1993) The macrofungus flora of China's Guangdong Province. The Chinese University Pres, Hong Kong.

17 Yang ZL, Wang XH, Binder M (2003) A study of the type and additional materials of Boletus thibetanus. Mycotaxon 86: 283-290.

18 Yang ZL (2005) Diversity and biogeography of higher fungi in China. Evolutionary genetics of fungi Horizon Bioscience. Norfolk 35-62.

19 Gelardi M, Vizzini A, Ercole E, Horak E, Ming Z, et al. (2015) Circumscription and taxonomic arrangement of Nigroboletus roseonigrescens gen. et sp. nov., a new member of Boletaceae from tropical South-Eastern China. PLoS ONE e0134295.

20 Li YC, Feng B, Yang ZL (2011) Zangia, a new genus of Boletaceae supported by molecular and morphological evidence. Fungal Diversity 49(1): 125143.

21 Li YC, Li F, Zeng NK, Cui YY, Yang ZL (2014) A new genus Pseudoaustroboletus (Boletaceae, Boletales) from Asia as inferred from molecular and morphological data. Mycological Progress 13: 1011.

22 Li YC, Ortiz-Santana B, Zeng NK, Feng B, Yang ZL (2014) Molecular phylogeny and taxonomy of the genus Veloporphyrellus. Mycologia 106(2): 291-306.

23 Li TH, Song B, Shen YH (2002) A new species of Tylopilus from Guangdong. Mycosystema 21(1): 3-5.

24 Feng B, Xu J, Wu G, Hosen MI, Zeng NK, et al. (2012) DNA sequence analyses reveal abundant diversity, endemism and evidence for Asian origin of the porcini mushrooms. PLoS ONE 7: e37567.

25 Zhao K, Wu G, Feng B, Yang ZL (2014a) Molecular phylogeny of Caloboletus (Boletaceae) and a new species in East Asia. Mycological Progress 13: 1001.

26 Zhao K, Wu G, Yang ZL (2014b) A new genus, Rubroboletus, to accommodate Boletus sinicus and its allies. Phytotaxa 188(2): 61-77.

27 Zhang M, Li TH, Bau T, Song B (2012) A new species of Xerocomus from Southern China. Mycotaxon 121: 23-27.

28 Zhang M, Li TH, Song B (2014) A new slender species of Aureoboletus from southern China. Mycotaxon 128: 195-202. 


\section{Open Access Journal of Mycology \& Mycological Sciences}

29 Zhang M, Li TH, Wang CQ, Song B, Xu J (2015a) Aureoboletus formosus, a new bolete species from Hunan Province of China. Mycological Progress 14: 118.

30 Zhang M, Li TH, Xu J, Song B (2015b) A new violet brown Aureoboletus (Boletaceae) from Guangdong of China. Mycoscience 56(5): 481-485.

31 Zeng NK, Cai Q, Yang ZL (2012) Corneroboletus, a new genus to accommodate the southeast Asian Boletus indecorus. Mycologia 104(6): 1420-1432.

32 Zeng NK, Tang LP, Li YC, Tolgor B, Zhao Q, et al. (2013) The genus Phylloporus (Boletaceae, Boletales) from China: morphological and multilocus DNA sequence analyses. Fungal Diversity 58(1): 73-101.

33 Zeng NK, Liang ZQ, Yang ZL (2014) Boletus orientialbus, a new species with white basidioma from subtropical China. Mycoscience 55(3): 159-163.

34 Zeng NK, Wu G, Li YC, Liang ZQ, Yang ZL (2014) Crocinoboletus, a new genus of Boletaceae (Boletales) with unusual boletocrocin polyene pigments. Phytotaxa 175(3): 133-140.

35 Zeng NK, Su MS, Liang ZQ, Yang ZL (2015) A geographical extension of the North American genus Bothia (Boletaceae, Boletales) to East Asia with a new species B. fujianensis from China. Mycological Progress 14: 1015.

36 Zeng NK, Chai H, Jiang S, Xue R, Wang Y, et al. (2018) Retiboletus nigrogriseus and Tengioboletus fujianensis, two new boletes from the south of China. Phytotaxa 367(1): 45-54.

37 Zhu XT, Li YC, Wu G, Feng B, Zhao K, et al. (2014) The genus Imleria (Boletaceae) in East Asia. Phytotaxa 191(1): 81-98.

38 Zhu XT, Wu G, Zhao K, Halling RE, Yang ZL (2015) Hourangia, a new genus of Boletaceae to accommodate Xerocomus cheoi and its allied species. Mycological Progress 14(6): 37.

39 Cui YY, Feng B, Wu G, Xu J, Yang ZL (2016) Porcini mushrooms (Boletus sect. Boletus) from China. Fungal Diversity 81(1): 189-212.

$40 \mathrm{Wu}$ G, Feng B, Xu J, Zhu XT, Li YC, et al. (2014) Molecular phylogenetic analyses redefine seven major clades and reveal 22 new generic clades in the fungal family Boletaceae. Fungal Diversity 69(1): 93115.

41 Wu G, Li YC, Zhu XT, Zhao K, Han LH, et al. (2016) One hundred noteworthy boletes from China. Fungal Diversity 81: 25-188.

42 Wu G, Zhao K, Li YC, Zeng NK, Feng B, et al. (2016) Four new genera of the fungal family Boletaceae. Fungal Diversity 81(1): 1-24.

43 Hosen MI, Li TH, Li T, Zhong XJ, Chen Y (2016) Tricholoma sinoacerbum, a bitter species from Guangdong Province of China. Mycoscience 57(4): 233-238.

44 Luo T], Zeng SZ, Deng C (2008) Xiangtoushan nature reserve (in Chinese). Chinese Literature and History Publishing Press.

45 Takahashi $H$ (2007) Five new species of the Boletaceae from Japan. Mycoscience 48(2): 90-99.

46 Smith AH, Thiers HD (1971) Boletes of Michigan. University of Michigan Press.

47 Singer R (1986) The Agaricales in modern taxonomy. $4^{\text {th }}$ (Edn.), Koeltz Scienific Books, Koenigstein.

48 Halling RE, Nuhn M, Fechner NA, Osmundson TW, Soytong K, et al. (2012) Sutorius: a new genus for Boletus eximius. Mycologia 104(4): 951-961. 